\section{Kidney \\ Blood Pressure \\ Research}

Kidney Blood Press Res 2014;39:260-271

DOI: 10.1159/000355803

Publisned ontıne: August 09, 2014

C 2014 S. Karger AG, Basel

www.karger.com/kbr

Accepted: June 02, 2014

1423-0143/14/0394-0260\$39.50/0

This is an Open Access article licensed under the terms of the Creative Commons AttributionNonCommercial 3.0 Unported license (CC BY-NC) (www.karger.com/OA-license), applicable to the online version of the article only. Distribution permitted for non-commercial purposes only.

Review

\title{
Recent Advances on Hepatitis C Virus in Dialysis Population
}

\author{
Fabrizio Fabrizia, Piergiorgio Messa $^{a} \quad$ Paul Martin $^{b}$ \\ aDivision of Nephrology, Maggiore Hospital, IRCCS Foundation, Milano, Italy; ${ }^{b}$ Division of Hepatology, \\ School of Medicine, University of Miami, Florida, USA
}

\section{Key-Words}

Hepatitis C Virus • Dialysis • Cardiovascular diseases $\bullet$ Inflammation $•$ Nutrition

\begin{abstract}
Hepatitis $\mathrm{C}$ virus $(\mathrm{HCV})$ infection remains common among patients undergoing regular dialysis and good evidence supports the detrimental role of HCV on survival in patients undergoing maintenance dialysis. According to an updated meta-analysis of clinical studies ( $n=15 ; 195,370$ unique patients on maintenance dialysis), the summary estimate for adjusted relative risk (allcause mortality) with anti-HCV across the published studies was 1.32 with a 95\% Confidence Intervals of $1.24 ; 1.42$, homogeneity assumption was not rejected. Various mechanisms support the excess death risk of HCV-infected patients on regular dialysis, in addition to liver disease-related mortality. The adjusted relative risk for cardiovascular mortality among HCV-infected patients on regular dialysis was 1.26 (95\% Confidence Intervals, 1.10; 1.45); the increased cardiovascular mortality in anti-HCV positive patients has been associated in part to malnutrition and chronic inflammation. The current standard of care for HCV in dialysis population is combined antiviral therapy (pegylated interferon plus ribavirin) with a rate of viral response of around 60\%. Triple therapy with telaprevir proved to be effective and safe in dialysis patients with HCV but only anecdotal evidence exists. Antiviral treatment of $\mathrm{HCV}$-infected patients on maintenance dialysis could lead to cure the liver damage and the extrahepatic complications. The future availability of all-oral interferon/ribavirin free regimens for antiviral treatment of HCV will help nephrologists to improve survival in this high-risk group.
\end{abstract}

Copyright $@ 2014$ S. Karger AG, Basel

\section{Introduction}

The estimated prevalence of HCV is 3\% in the world population (around 170 million infected individuals worldwide) heavily burdens public health [1]. HCV continues to be common among patients undergoing maintenance dialysis all over the world despite the 


\section{Kidney \\ Blood Pressure Research}

advent of serologic screening of blood by enzyme-linked immunoassays, routine use of erythropoietin for patients with anemia and chronic kidney disease, and improved compliance with infection control procedures to prevent the spread of hepatitis $\mathrm{C}$ virus within dialysis units [2].

HCV infection remains the most frequently recognized cause of liver damage in chronic kidney disease (CKD) patients [3, 4]. Evidence accumulated in the last decade shows a link between HCV and reduced survival in patients on maintenance dialysis; the mechanisms underlying this relationship are an area of active research. In addition to liver disease-related mortality risk, an impairment of health-related quality of life [5] and a greater cardiovascular risk have been implicated among HCV-infected patients on maintenance dialysis [6]. HCV has also been recognized as an independent risk factor for cardiovascular disease in the adult general population [7].

On the other hand, a recent survey from the Dialysis Outcomes and Practice Patterns Study (DOPPS) has demonstrated that HCV-infected patients on maintenance hemodialysis very rarely receive antiviral therapy based on interferon (IFN) [8]. It is likely that the future availability of newer, all oral regimens with high rates of viral response will promote the antiviral treatment of HCV in these 'difficult to treat' patients. The aim of this review is to report the most recent discoveries regarding natural history and antiviral treatment of HCV infection among patients undergoing regular dialysis.

\section{Natural history of HCV in dialysis patients}

HCV infection remains common in patients undergoing long-term dialysis both in developed and less-developed world. A survey on 8,615 patients from the DOPPS yielded a mean prevalence of $13.5 \%$ (2.6 to $22.9 \%$ ) across seven developed countries including UK and the US [9]. The natural history of HCV infection in patients on long-term dialysis is not completely understood, and this is probably related to several factors. HCV infection in dialysis patients is usually asymptomatic with an apparent indolent course. Because the natural history of HCV infection extends over decades rather than years even in patients with intact kidney function, adverse consequences of chronic HCV infection may not be obvious in patients followed for shorter periods of time. Patients with chronic kidney disease have higher morbidity and mortality rates than the general population because their average age is greater and because of common comorbidities.

Biochemical evaluation of HCV infection in patients with chronic kidney disease is commonly unaccurate. HCV infection results in increased serum alanine aminotransferase levels even among patients on maintenance dialysis but aminotransferase activity is lower in dialysis than nonuremic patients and this hampers the recognition of HCV-related liver disease on the grounds of biochemical tests [10]. Clinicians are commonly reluctant to perform liver biopsies in dialysis patients because of concern about platelet function in chronic uremia. The recent implementation of guidelines recommending antiviral therapy for HCV infection in patients with chronic kidney disease makes difficult the conduction of longitudinal studies on the natural history of HCV infection in patients with CKD [11].

Some investigators have suggested a milder course of HCV infection among patients on maintenance dialysis [12], and this has been attributed to the immune compromise conferred from chronic uraemia. Immunologic abnormalities which are the predominant pathophysiologic mechanism responsible for hepatocyte injury in HCV-infected patients are lowered among patients on longt-term dialysis. An exaggerated synthesis of cytokines including hepatocyte growth factor [12], tumor necrosis factor- $\alpha$, interleukin-1 $\beta$, and interleukin-6 [13] during the haemodialysis session has been hypothesized. 


\section{Kidney \\ Blood Pressure Research}

\section{HCV and survival in dialysis population}

Mortality is an identifiable complication of liver disease and a reliable end-point in the natural history of HCV-related liver disease. Data concerning the relationship between HCV infection and risk for death among HCV-infected patients on long-term dialysis are not abundant. However, longitudinal studies with appropriate size and follow-up have found an independ-

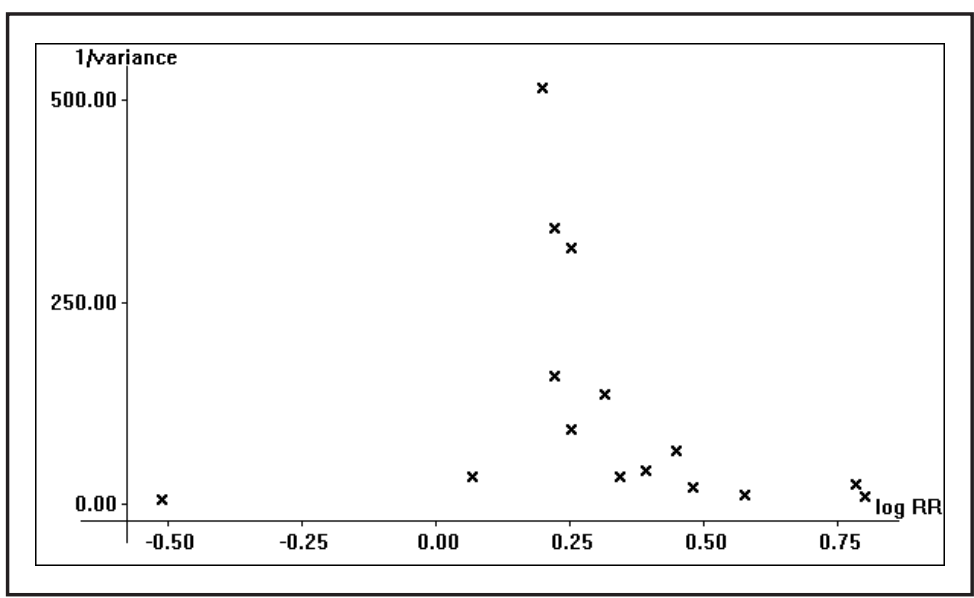

Fig. 1. Meta-analysis on HCV infection and survival in dialysis patients: Funnel plot.

ent and significant relationship between anti-HCV positive serologic status and lower patient survival. According to an updated meta-analysis of observational studies (fourteen papers giving information on fifteen clinical studies; $n=195,370$ unique patients on longterm dialysis), anti-HCV antibody status was an independent and significant risk factor for death in individuals receiving maintenance dialysis. The summary estimate for adjusted relative risk (aRR)(all-cause mortality) was 1.32 with a 95\% CI of 1.24; 1.42 . Tests for homogeneity of the aRR across the fifteen studies gave Ri value of 0.37 , that is, the homogeneity assumption was not rejected (Figure 1). Stratified analysis showed that the adjusted relative risk of liver disease-related death was 3.82 (95\% CI, 1.92; 7.61); the excess risk of liverrelated death in HCV-positive patients may be at least attributed to chronic liver disease with its attendant complications, cirrhosis and hepatocellular carcinoma (HCC) [6].

We cannot rule out an underestimation of negative results related to publication bias (positive relationships published more than negative ones); in addition, the potential for additional confounders cannot be excluded. On the other hand, the detrimental role of HCV on survival in dialysis patients had been observed from other sources. Butt et al. evaluated a cohort of 16,965 patients on manteinance dialysis from the 1997-1998 United States Renal Data System, 5,737 being HCV-infected. HCV infection was associated with increased risk of mortality over time $(P<0.0001)$ [14]. Goodkin et al. reported on the DOPPS survey enrolling 49,762 patients receiving maintenance haemodialysis in 12 countries between 1996 and 2011. 4,735 (9.5\%) patients with HCV infection were identified; in the subset of HCV positive patients with propensity for antiviral treatment, the hazard ratio for adjusted all-cause mortality was lower in treated compared with untreated patients even if no difference was found $(0.47 ; 95 \% \mathrm{CI}, 0.17 ; 1.26)$ (NS). Four $(9.5 \%)$ of 42 treated HCV positive patients and $638(21 \%)$ of 3,037 untreated HCV-infected patients on maintenance dialysis died (median follow-up of 1.4 years per study phase) [8].

\section{HCV and cardiovascular mortality risk in dialysis population}

In addition to the link between HCV infection and all-cause mortality in dialysis population, recent studies have addressed the relationship between positive anti-HCV serologic status and disease-specific mortality in patients on dialysis. It remains unclear whether the increased mortality risk in HCV-positive patients on maintenance dialysis is related only to a higher rate of cirrhosis and HCC which are common comorbidities in HCV-infected patients on dialysis. Henderson et al. identified a large cohort of HCV-infected 


\section{Kidney Blood Pressure Research}

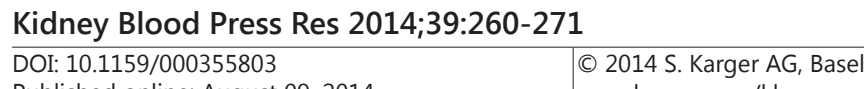

Publisnea onine: August 09, 2014 www.karger.com/kbr

Fabrizi/Messa/Martin: HCV in Dialysis

patients $(n=32,806)$ on maintenance dialysis using ICD-9 codes of the United States Renal Data System; the frequency of cirrhosis $(n=3,765)$ and HCC $(n=262)$ was $11.5 \%$ and $0.79 \%$, respectively. The most important predictor of occurrence of HCC being cirrhosis (OR, 11.87; 95\% CI, 8.8; 15.5, $P<0.001$ ) [15].

Other reasons for the increased death risk in HCV-positive patients on long-term dialysis include co-infection with hepatitis B virus (HBV) or human immunodeficiency virus (HIV), impaired quality of life, and cardiovascular diseases. According to our meta-analysis of clinical studies, the adjusted RR of cardiovascular mortality in dialysis patients was 1.26 $(95 \% \mathrm{CI}, 1.10 ; 1.45)(P<0.01)[6]$. Only three reports of our meta-analysis of observational studies gave detailed information on this point; however, no heterogeneity was observed (NS) and the subset of studies was large $(n=37,789)$. That the most important cause of death in patients on long-term dialysis is cardiovascular is well known from the 1990s. However, we observed the persistence of an increased HCV-associated cardiovascular risk after adjustment for several covariates including age, gender, dialysis vintage, diabetes mellitus, and others. In addition to conventional risk factors for cardiovascular mortality in dialysis patients such as arterial hypertension, hypercholesterolemia, hyper-homocysteinemia, HCV infection may be an additional factor. This opinion is promoted by the notion that traditional risk factors only partially explain the mortality excess among patients on maintenance dialysis.

Some lines of evidence are in accordance with a link between anti-HCV positive serologic status and cardiovascular mortality among patients undergoing maintenance dialysis. Kalantar-Zadeh and colleagues were the first investigators to establish a greater cardiovascular risk in anti-HCV positive patients on regular dialysis, according to their population-based survey $(2,778$ patients on maintenance dialysis at DaVita dialysis clinics across the US), 13\% $(n=363)$ being anti-HCV positive [16]. A relationship between antiHCV positive serologic status and cardiovascular mortality in the subset of dialysis patients younger than 65 years $(n=1,551)$ was found, after adjustment for case-mix and available surrogates for malnutrition-inflammation complex syndrome, $\mathrm{HR}=1.80$ (95\% CI, 1.1; 2.95, $P=0.02)$. Bose et al. analyzed a large cohort of HCV-infected individuals $(n=424)$ treated with haemodialysis $(n=290)$ and peritoneal dialysis $(n=134)$ in Australia and New Zealand over the period 1994-2008, and found that coronary artery disease (CAD) was an independent risk factor for mortality, 1.75 (1.34 to 2.30), $P<0.01$ [17]. Butt et al. identified 5,310 patients with HCV infection on long-term dialysis in the US using ICD-9 codes (United States Renal Data System); in addition to increasing age, diabetes mellitus, cirrhosis, wasting, and cancer, coronary artery disease $(1.81 ; 95 \% \mathrm{CI}, 1.57 ; 2.08, P<0.001)$ was associated with higher odds of death [18]. Roth et al. perfomed a single-center analysis of clinical outcomes over approximately 20 years in 230 consecutive patients determined to be HCV-infected during their pretransplant evaluation. They observed a positive and consistent influence of kidney transplantation on the risk of cardiovascular mortality, the impact of renal transplantation upon the hazard risk of death caused by a cardiovascular event $(n=175 ; 32$ deaths: 19 pretransplant and 13 post-transplant) was 0.20 (95\% CI, 0.08; 0.47) $(P=0.0002)$. The favourable role of renal transplantation on all-cause mortality beyond 6 months after transplant was explained by Roth et al. with a significantly lower cardiovascular death rate that occurs early after transplant and remains consistent over time [19].

\section{HCV and cardiovascular diseases in the general population: An update}

Several clinical studies performed in the adult general population have shown that certain metabolic disorders such as type 2 diabetes mellitus, insulin resistance, and hepatic steatosis have greater prevalence in patients with chronic HCV compared with non-infected patients. These features are relevant risk factors for cardiovascular morbidity and mortality in patients with intact kidney function and the ability of HCV to directly and indirectly interfere with glucose and lipid metabolism could take account into their pathogenesis. It has 


\section{Kidney Blood Pressure Research}

been suggested that HCV induces insulin resistance by downregulation of insulin receptors and beta-cell dysfunction [20]; insulin resistance may have several clinical consequences such as accelerated progression of liver fibrosis, resistance to antiviral therapy, development of hepatocellular carcinoma and perhaps atherosclerosis [21].

Whether HCV infection is associated with an increased cardiovascular risk in the adult genral population is an issue of active research. Cross-sectional data show that HCV positive patients have a $2.4 \%$ higher risk for cardiovascular disease, as assessed by the Framingham Risk Score, than the adult general population $(P<0.01)$. This was observed after controlling for several clinical or background parameters such as weight, marital status, current medications, and others [22]. To the best of our knowledge, the strongest evidence of a potential link between HCV infection and cardiovascular mortality emerged from longitudinal studies addressing the natural history of patients with chronic HCV and intact kidney function. The first work was of Guiltinian et al. who performed a retrospective cohort study on 10,259 anti-HCV positive and 10,259 age- and gender- matched anti-HCV negative US blood donors, followed from 1991 to 2002, and with a mean follow-up of 7.7 years. The HCV positive group had higher mortality (HR, 3.13; 95\% CI, 2.6; 3.76), and the excess mortality was greatest in liver -related, drug- or alcohol-related and trauma/ suicide causes. There was also an unexpected increase in cardiovascular mortality among the HCV positive donors, HR=2.21; 95\% CI, 1.41; 3.46 [23]. Three additional retrospective studies observed a higher risk of incidental coronary artery disease [24], acute coronary syndrome [25], and stroke [26] in HCV infected compared with HCV negative individuals. However, these data were not confirmed by Forde et al. in a large cohort of adults followed in general practices in the UK [27]. Butt et al. [24] used a national, observational cohort of HCV-infected veterans receiving care at all Veteran Affairs facilities, the Electronic Retrieved Cohort of HCV Infected Veterans to identify HCV-positive and -negative control subjects. They enrolled 82,083 HCV positive and 89,582 HCV negative patients and found that the incidence of coronary artery disease was higher in HCV -infected than HCV negative patients (HR, 1.25; 95\% CI, 1.20; 1.30), despite a more favourable metabolic profile in the first group. We retrieved three prospective studies which found an increased risk of stroke [28], lethal cerebrovascular events [29], and circulatory diseases [30] among HCV positive compared to -negative individuals with intact kidney function.

The potential association between HCV and cardiovascular diseases has been also explored with case control studies, which provide weaker epidemiologic evidence than prospective studies. At least five case control studies have evaluated the link between HCV infection and carotid atherosclerosis- most of these found a higher prevalence of carotid plaques in HCV infected patients compared with controls [7,31-35]. A few case control studies observed a significant relationship between HCV infection and coronary atherosclerosis [36, 37]. Finally, two independent groups found a strong association between HCV infection and myocardial dysfunction, defined as congestive heart failure or abnormal severity score by myocardial scintigraphy [38, 39].

The reasons for some conflicting results remain unknown. Discrepancies could likely be due to the clinical or demographic characteristics of the studied populations, or differences in the assessment of HCV infection (or its severity) or in the evaluation of carotid or coronary atherosclerosis. The potential confounding by unmeasured or inadequately controlled variables cannot be excluded. Overall, the available evidence suggests an excess risk of cardiovascular diseases among HCV positive patients in individuals with intact kidney function.

\section{HCV and cardiovascular diseases in dialysis patients: novel evidence}

In addition to the information reported above, novel investigative techniques are in keeping with a relationship between HCV infection and cardiovascular diseases. Yelken et al. [40] measured coronary flow reserve (CFR) by transthoracic Doppler echocardiography 


\section{Kidney Blood Pressure Research}

in nondiabetic anti-HCV positive $(n=26)$ and -negative $(n=26)$ patients undergoing regular dialysis (haemodialysis or peritoneal dialysis) after kidney transplant failure. Clinical and demographic characteristics were similar between the two groups. CFR was significantly lower in HCV-infected than uninfected individuals $(1.5 \pm 1.1$ vs. $1.63 \pm 0.26, P=0.03)$.

Oyake and colleagues [41] evaluated 94 dialysis patients (17 being anti-HCV positive/ HCV RNA positive) by prospective measurements of aortic stiffness using carotid-femoral pulse wave velocity (PWV). They found by multiple logistic regression analysis that mean blood pressure and $\mathrm{HCV}$ viremia $(\mathrm{OR}, 9.7 ; 95 \% \mathrm{CI}, 1.18 ; 81.2, P<0.05)$ were significantly and independently associated with high aortic PWV ( $>$ or $=10.0 \mathrm{~m} / \mathrm{sec}$, mean). During the followup period, 13 patients suffered from cardiovascular events. Kaplan-Meier survival curves for cerebrovascular and cardiovascular event-free rates indicated a significant difference between HCV-RNA positive and negative patients on dialysis (log-rank test, $P<0.05$ ). The authors mentioned an atherogenic role of HCV through aggravation of metabolic syndrome and dyslipidemia.

Conversely, Caliskan et al. [42] evaluated a cohort of 72 patients on maintenance hemodialysis and found no difference in total insulin secretion evaluated by HOMA- $\beta$ $(721 \pm 6221$ vs. $357 \pm 862 \mathrm{mIU} / \mathrm{mL}, P=0.76)$ and insulin resistance analyzed by HOMA-R $\left(2.41 \pm 3.0\right.$ vs. $\left.2.33 \pm 1.83 \mathrm{mIU} / \mathrm{mmol}^{-2}, P=0.91\right)$ between anti-HCV positive and -negative patients. The flow-mediated endothelium-dependent dilatation of the brachial artery measured by high resolution ultrasonography did not change between anti-HCV positive and -negative patients, $9.6 \pm 6.7 \%$ vs. $12 \pm 7.1 \%(P=0.17)$. Also, total cholesterol $(161 \pm 35 v s$. $189 \pm 59 \mathrm{mg} / \mathrm{dL}, P=0.019)$ and $\mathrm{LDL}$ cholesterol $(85 \pm 29$ vs. $109 \pm 47 \mathrm{mg} / \mathrm{dL}, P=0.012)$ were lower in anti-HCV positive than -negative patients on maintenance dialysis. They concluded that the impact of HCV on the cardiovascular risk is not evident in dialysis patients who have specific risk factors for cardiovascular mortality such as hyper-homocysteinemia, inflammation, or abnormalities in mineral metabolism. The impact of these factors probably overcomes the effect of HCV in this high-risk group.

\section{HCV and malnutrition-inflammation complex syndrome (MICS) in dialysis}

A key finding in the study by Kalantar-Zadeh et al. was that the short-term all-cause mortality (hazard ratio of 3-yr) was greater among virtually all subgroups of HCV-infected patients on maintenance dialysis [43]. In addition, they observed that HCV was more strongly associated with death in incident (vintage $<6$ months) (HR, 1.42; 95\% CI, 1.19; 1.69 , $P<0.001$ ) than in prevalent subgroups (vintage $\geq 6$ months) (HR, 1.18, 95\% CI, 1.02; 1.35, $P<0.02$ ). Thus, it is less likely that the HCV-associated death risk in dialysis patients is only due to such long-term complications as liver disease. It has been speculated that patients on maintenance hemodialysis show malnutrition-inflammation complex syndrome that may be exacerbated by chronic HCV and contribute to the excess risk of cardiovascular mortality among HCV positive patients. Tsai et al. performed a prospective, longitudinal study on 153 patients on maintenance dialysis in Taiwan (58 HCV positive and 95 -negative patients) [44]. The total malnutrition-inflammation score was significantly greater in patients with active HCV infection than non-HCV individuals at baseline $(P=0.034)$ and at last day of follow-up $(P=0.009)$. Kalantar-Zadeh et al observed that after adjustment for 13 available surrogates of MICS, the association between HCV infection and mortality was reduced in some subpopulations, as an example, the significant link between all-cause mortality and HCV was lost in patients aged 65 or older, or in patients with dialysis vintage $\geq 60$ months [43]. This may indicate that MICS is either a confounder or may be at least partially in the causal pathway. Elsurer et al. [45] conducted a cross-sectional single-center study in 22 anti-HCV positive HD patients with CAD and 61 anti-HCV positive HD patients without CAD (controls); they observed that malnutrition inflammation score (MIS) of anti-HCV positive patients with CAD was greater than in patients without CAD, $8.8 \pm 4.0$ vs. $6.5 \pm 2.6, P=0.02$. A multivariate stepwise backward logistic regression analysis of factors associated with 


\section{Kidney Blood Pressure Research}

coronary artery disease found that_the malnutrition inflammation score was significantly related to CAD, OR, 1.27 (95\% CI, 1.008; 1.6; $P=0.043$ ) in anti-HCV positive patients on maintenance hemodialysis.

Various pieces of evidence support a link between HCV and low nutritional status among patients undergoing maintenance dialysis, irrespective of cirrhotic status. According to Tsai et al., active HCV infection was associated with low cholesterol levels, OR, 0.98 (95\% CI, 0.98; 0.99, $P<0.016$ ] [44]. Kalantar-Zadeh et al. suggested that each $0.2 \mathrm{gr} / \mathrm{dL}$ decrease in serum albumin was associated with a 1.40 -fold increased OR of active HCV infection $(95 \%$ CI, 1.31;1.50, $P<0.01$ ] [16]. Caliskan and colleagues found that total and LDL-cholesterol levels were lower in anti-HCV positive than -negative patients on maintenance hemodialysis, $161 \pm 35$ vs. $189 \pm 59 \mathrm{mg} / \mathrm{dL}(P=0.019)$ and $85 \pm 29 v s .109 \pm 47 \mathrm{mg} / \mathrm{dL}(P=0.012)$ [42]. In their cross-sectional study including HCV positive patients with intact kidney function $(n=159)$ and end-stage renal disease $(n=91)$, Hu et al. found by multivariate analysis that chronic hepatitis $\mathrm{C}$ and end-stage renal disease were independently associated with higher frequency of hypoalbuminemia (OR, 9.56; 95\% CI, $P=0.0007$ ) [46]. Wang et al. retrospectively reviewed 538 patients on peritoneal dialysis, $75(13.9 \%)$ being anti-HCV positive; serum levels of albumin were significantly lower in anti-HCV positive than -negative patients $(3.1 \pm 1 \mathrm{vs}$. $3.4 \pm 0.8 \mathrm{mg} / \mathrm{dL})(P<0.05)$. Also, cholesterol $(178 \pm 44 v s .198 \pm 52 \mathrm{mg} / \mathrm{dL}, \mathrm{NS})$ and trygliceride $(194 \pm 121$ vs. $212 \pm 174 \mathrm{mg} / \mathrm{dL}, \mathrm{NS})$ levels were lower in anti-HCV positive patients even if no significance was reached [47]. Thus, a detrimental impact of HCV infection on nutritional status might result in increased mortality among dialysis patients. HCV infection has been also associated in dialysis patients with some markers of systemic inflammation such as C-reactive protein, interleukin-6, interleukin-1 and tumour necrosis factor- $\alpha$. Nascimento et al. enrolled a total of 115 patients undergoing maintenance haemodialysis at three dialysis centers in Brazil; 62 (55\%) being anti-HCV positive [48]. Plasma pentosidine levels were greater in HCV-positive than-negative patients, 105 (13-233) vs. 87 (26-184) pmol/ mg albumin, $P=0.04$. In a stepwise backward multiple regression model, age and HCV were independent predictors of pentosidine levels $\left(P<0.05, r^{2}=0.07\right)$, possibly reflecting increased oxidative stress. Yelken et al. noted that serum high-sensitivity C-reactive protein levels, well-known markers of inflammation, were significantly greater in HCV positive patients than HCV negative counterparts, $16 \pm 14.9$ vs. $7.2 \pm 5.9 \mathrm{mg} / \mathrm{dl}, P=0.012(n=52$ dialysis patients with failed renal allografts) [40]. They concluded that graft dysfunction per se supports endothelial dysfunction and HCV is another trigger of endothelial damage. In a separate report, Nascimento and coworkers found that the ratio between plasma high sensitivity C-reactive protein and interleukin-6 (hsCRP/IL-6) was significantly lower in anti-HCV positive than -negative patients, $0.9 \pm 0.8$ vs. $1.8 \pm 3.2, P<0.05$ [49].

Of note, conflicting data have been given also on this point. As an example, Arsov et al. measured advanced glycation end products (AGE) accumulation by means of skin autofluorescence in patients receving regular hemodialysis [50]. They found a greater accumulation of advanced glycation end products in patients on regular HD $(n=92)$ than in age-matched controls $(n=93), 3.13 \pm 0.95$ vs. $2.20 \pm 0.47$ AU; $P<0.001$. Multivariate analysis showed that AGE accumulation in patients on intermittent HD is linked to an independent effect of age $(P<0.05)$, diabetes mellitus $(P<0.01)$, time on dialysis $(P=0.03)$. No significant difference occurred between skin autofluorescence values of HCV positive and -negative patients on HD, $3.20 \pm 0.96$ vs. $3.03 \pm 0.96 \mathrm{AU}, P=0.39$. The conclusion of these authors was that an identical level of oxidative stress between HCV positive and -negative patients on regular HD exists.

\section{Anti-viral therapy of HCV in dialysis population: new rationale?}

Available evidence indicates that HCV-infected kidney recipients have worse patient and allograft survival after transplantation compared to uninfected kidney transplant recipients [11]. In fact, by virtue of their mechanisms of action, immunosuppressive therapies show 


\section{Kidney Blood Pressure Research}

Kidney Blood Press Res 2014;39:260-271

\begin{tabular}{l|l}
\hline DOI: 10.1159/000355803 & (C) 2014 S. Karger AG, Basel
\end{tabular}

Publisned onitne: August 09, 2014

www.karger.com/kbr

the potential to have a permissive effect on HCV viral replication after transplantation. This may result in an accelerated and severe course of HCV-associated liver disease. Several extra-hepatic complications of HCV post-transplant have been observed including new onset diabetes mellitus, de novo or recurrent glomerulonephritis, chronic allograft nephropathy ${ }_{2}$ and sepsis. In addition, reduced drug clearance in the setting of hepatic dysfunction may affect blood levels of commonly used immunosuppressive agents that are metabolized in the liver, such as calcineurin inhibitors [11]. To avoid these outcomes, treatment of HCV is strongly recommended in renal transplant candidates, dialysis-dependent or not; in fact, IFNbased regimens are contraindicated after kidney transplantation due to the increased risk of allograft dysfunction. KDIGO HCV guidelines suggest IFN-based therapy only for kidney transplant recipients with hepatitis $C$ in whom the benefits of treatment clearly outweigh the risks (cholestatic fibrosing hepatitis, severe vasculitis, rapid cirrhosis) [11]. The most important benefit of successful antiviral therapy in HCV-infected patients on long-term dialysis includes slowing the progression of liver disease. The link between cardiovascular mortality, MICS, and HCV seropositive status among patients on maintenance dialysis, if conclusively confirmed, could lead to treatment of HCV infection not only to repair liver damage but also to lower cardiovascular and extra-hepatic complications. The availability in the near future of anti-HCV agents provided with high safety and efficacy should help nephrologists to improve survival of patients on long-term dialysis.

\section{Current standard of care of antiviral therapy of HCV in dialysis population}

A good number of clinical studies have shown that approximately one third of patients with chronic HCV receiving regular dialysis achieve sustained virologic response by conventional or pegylated interferon monotherapy. Ribavirin had been considered to be contraindicated in patients with HCV undergoing regular dialysis because of concern for lifethreatening anemia. Evidence has been accumulated over the last decade showing that antiviral combination therapy is feasible in dialysis population by adding low-dose ribavirin (200 $\mathrm{mg}$ three times weekly to $400 \mathrm{mg}$ daily) $[11,51]$. Thus, low-dose ribavirin has been approved (August 2011) in the US for HCV therapy in individuals on long-term hemodialysis. We have performed a meta-analysis of observational studies (287 unique patients, $n=11$ clinical studies) and calculated that the summary estimate for sustained virological response (SVR) after combination antiviral therapy was 0.60 (95\% Confidence Intervals, $0.47 ; 0.71$ ). The summary estimate for drop-out rate was 0.18 (95\% CI, 0.08 ; 0.35$)$, the most common source of drop-outs being anemia $(11 / 46=23 \%)$ [52]. According to these findings, combination antiviral therapy (pegylated interferon and low dose ribavirin) should be considered the current standard of care for HCV-infected patients on regular dialysis.

In order to improve efficacy and safety of antiviral therapy towards HCV, two distinct classes of hepatitis $\mathrm{C}$ antiviral agents, direct-acting antivirals (DAAs) and host-targeting antivirals (HTAs) have impacted the HCV field by giving higher sustained viral responses rates, reducing adverse side effects and shortening the treatment duration. Some DAAs have been recently included into the standard of care, others are currently incorporated in the most advanced clinical trials. All the major HCV-induced enzymes, namely, NS2-3 and NS34A proteases, NS3 helicase, and NS5B RNA-dependent RNA polymerase are crucial for HCV replication and are potential drug discovery targets (Figure 2) [53, 54].

\section{Antiviral therapy of HCV in dialysis and DAAs and host-targeting agents}

The first examples of DAAs are boceprevir (BOC) and telaprevir (TVR) [53]. They are classified as 'first-wave' NS3/4A inhibitors and are the first examples of DAAs; in association with pegylated/ribavirin combination they increased SVR rates by about $30 \%$ and $25-60 \%$ in treatment-naïve and treatment-experienced patients, respectively. At the present time, 


\section{Kidney \\ Blood Pressure \\ Research}

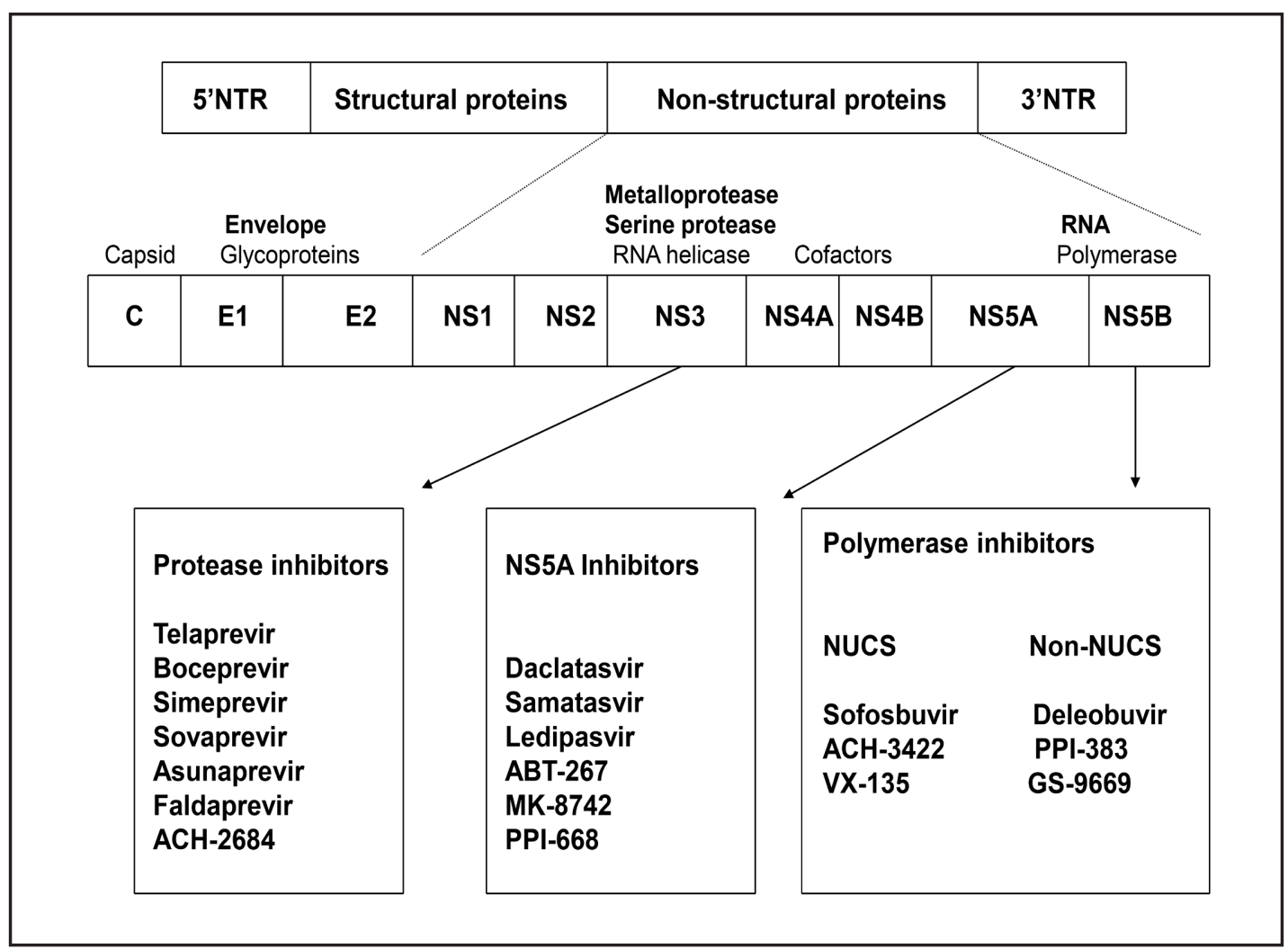

Fig. 2. Potential drug discovery targets for hepatitis $\mathrm{C}$ virus (HCV) and $\mathrm{HCV}$ genome.

combination triple therapy with pegylated interferon, ribavirin and first-generation protease inhibitors is the SoC for chronic genotype $1 \mathrm{HCV}$ infection.

The information on the antiviral therapy of HCV in dialysis population in the era of DAAs is scarce. Dumortier et al. [55] addressed efficacy and tolerability of available protease inhibitors in HCV-infected haemodialysis patients. Four HCV-infected patients (HCV genotype 1b) on long-term haemodialysis and listed for kidney transplant, who have not responded to a prior course of pegylated interferon and ribavirin received triple therapy with telaprevir. After 12 weeks of triple therapy, HCV RNA became undetectable in 3 out of 4 (75\%) patients; ribavirin dose ranged from $200 \mathrm{mg}$ three times a week to $200 \mathrm{mg} /$ day. The patients tolerated treatment well and did not present significant side effects, except moderate asthenia and anaemia. Erythropoietin (EPO) was started in the first patient and increased EPO doses were used by the others. It is clear that these results need to be confirmed- the size of the study group was small and the follow-up was short. In addition, the authors suggested longitudinal through plasma measurements to show that no telaprevir accumulation occurs after steady state is reached. No modification of any concomitant medication was made.

\section{Conclusions}

Hepatitis $\mathrm{C}$ virus infection is common among patients with chronic kidney disease on long-term dialysis and is an important risk factor for mortality in this population. In addition to the conventional risk factors for cardiovascular diseases in dialysis patients, HCV may be an additional factor. Combination antiviral therapy (pegylated interferon plus ribavirin) should be considered the current standard of care for HCV among patients on maintenance 


\section{Kidney \\ Blood Pressure Research}

dialysis. The evidence on triple therapy with telaprevir for HCV in dialysis population is extremely limited. Prospective clinical trials are under way to define antiviral combination regimens provided with high rates of viral eradication and minimal side-effects in dialysis population.

\section{Disclosure Statement}

The authors of this manuscript state that they do not have any conflict of interests and nothing to disclose.

\section{References}

1 Pol S, Vallet-Pichard A, Corouge M, Mallet V: Hepatitis C: Epidemiology, diagnosis, natural history and therapy. Contrib Nephrol 2012;176:1-9.

-2 Jadoul M, Barril G: Hepatitis C in hemodialysis: Epidemiology and prevention of hepatitis C virus transmission. Contrib Nephrol 2012;176:35-41.

3 Ozdogan M, Ozgur O, Gur G, Boyacioglu S, Ozderin Y, Demirhan B, Telatar H, Haberal M: Histopathological impact of hepatitis virus infection in hemodialysis patients: should liver biopsy be performed before renal transplantation? Artif Organs 1997;21:355-358.

4 Perez R, Ferreira A, Silva I, Medina-Pestana J, Lanzoni V, Silva A, Ferraz M: Hepatitis C virus infection in renal transplant patients: a comparative study with immunocompetent patients. Clin Transplant 2005;19:763-768.

5 Fabrizi F, Messa P, Martin P: Health-related quality of life in dialysis patients with HCV infection. Int J Artif Organs 2009;32:473-481.

6 Fabrizi F, Dixit V, Messa P: Impact of hepatitis C on survival in dialysis patients: a link with cardiovascular mortality? J Viral Hepat 2012;19:601-607.

7 Petta S, Macaluso F, Craxi A: Cardiovascular diseases and HCV infection: a simple association or more? Gut 2014;63:369-375.

-8 Goodkin D, Bieber B, Gillespie B, Robinson B, Jadoul M: Hepatitis C infection is very rarely treated among hemodialysis patients. Am J Nephrol 2013;38:405-412.

-9 Fissell R, Bragg-Gresham J, Woods J, Jadoul M, Gillespie B, Hedderwick S, Rayner H, Greenwood R, Akiba T, Young E: Patterns of hepatitis C prevalence and seroconversion in hemodialysis units from three continents: the DOPPS. Kidney Int 2004;65:2335-2342.

-10 Fabrizi F, Martin P, Dixit V, Brezina M, Cole M, Gerosa S, Vinson S, Mousa M, Gitnick G: Quantitative assessment of HCV load in chronic haemodialysis patients: a cross-sectional survey. Nephron 1998;80:428433.

11 Kidney Disease: Improving Global Outcomes: KDIGO clinical practice guidelines for the prevention, diagnosis, evaluation and treatment of hepatitis C in chronic kidney disease. Kidney Int 2008;73:S1-S99.

12 Rampino T, Arbustini E, Gregorini M, Guallini P, Libetta C, Maggio M, Ranghino A, Silini E, Soccio G, Dal Canton A: Hemodialysis prevents liver disease caused by hepatitis $\mathrm{C}$ virus: role of hepatocyte growth factor. Kidney Int 1999;56:2286-2291.

13 Malaponte G, Bevelacqua V, Fatuzzo P, Rapisarda F, Emmanuele G, Travali S, Mazzarino M: IL-beta, TNFalpha and IL-6 release from monocytes in hemodialysis patients in relation to dialytic age. Nephrol Dial Transplant 2002;17:1964-1970.

14 Butt A, Skanderson M, McGinnis K, Ahuja T, Bryce C, Barnato A, Chang C: Impact of hepatitis C virus infection and other comorbidities on survival in patients on dialysis. J Viral Hepat 2007;14:688-696.

15 Henderson W, Shankar R, Gill J, Kim K, Ghany M, Skanderson M, Butt A: Hepatitis C progressing to hepatocellular carcinoma: the HCV dialysis patient in dilemma. J Viral Hepat 2010;17:59-64.

16 Kalantar-Zadeh K, McAllister C, Miller L: Clinical characteristics and mortality in hepatitis C-positive haemodialysis patients: a population based study. Nephrol Dial Transplant 2005;20:1662-1669. 


\section{Kidney \\ Blood Pressure Research}

17 Bose B, McDonald S, Hawley C, Brown F, Badve S, Wiggins K, Bannister K, Boudville N, Clayton P, Johnson D: Effect of dialysis modality on survival of hepatitis C-infected ESRF patients. Clin J Am Soc Nephrol 2011;6:2657-2661.

18 Butt A, Khan U, Skanderson M: Comorbidities and their impact on mortality in HCV and HCV-HIV coinfected persons in dialysis. J Clin Gastroenterol 2008;42:1054-1059.

19 Roth D, Gaynor J, Reddy K, Ciancio G, Sageshima J, Kupin W, Guerra G, Chen L, Burke G: Effect of kidney transplantation on outcomes among patients with hepatitis C. J Am Soc Nephrol 2011;22:1152-1160.

20 Shintani Y, Fujie H, Miyoshi H, Tsutsumi T, Tsukamoto K, Kimura S, Moriya K, Koike K: Hepatitis C virus infection and diabetes: direct involvement of the virus in the development of insulin resistance. Gastroenterology 2004;126:840-848.

-21 Mangia A, Ripoli M: Insulin resistance, steatosis, and hepatitis C virus. Hepatol Int 2013; 7:S782-S789.

22 Kakinami L, Block R, Adams M, Cohn S, Maliakkal B, Fisher S: Risk of cardiovascular disease in HIV, hepatitis C, or HIV/hepatitis C patients compared to the general population. Int J Clin Pract 2013;67:6-13.

23 Guiltinian A, Kaidarova Z, Custer B, Orland J, Strollo A, Cyrus S, Busch M, Murphy E: Increased allcause, liver, and cardiac mortality among hepatitis $\mathrm{C}$ virus-seropositive blood donors. Am J Epidemiol 2008;167:743-750.

24 Butt A, Xiaoqiang W, Budoff M, Leaf D, Kuller L, Justice A: Hepatitis C virus infection and the risk of coronary disease. Clin Infect Dis 2009;49:225-232.

25 Hsu Y, Lin J, Ho H, Kao Y, Huang Y, Hsiao N, Wu M, Liu Y, Wu C: Antiviral treatment for hepatitis C virus infection is associated with improved renal and cardiovascular outcomes in diabetic patients. Hepatology 2014;59:1293-1302.

26 Hsu C, Kao J, Chao Y, Lin H, Fan Y, Huang C, Tsai P: Interferon-based therapy reduces risk of stroke in chronic hepatitis $\mathrm{C}$ patients: a population-based cohort study in Taiwan. Aliment Pharmacol Ther 2013;38:415-423.

27 Forde K, Haynes K, Troxel A, Trooskin S, Osterman M, Kimmel S, Lewis J, Lo Re V: Risk of myocardial infarction associated with chronic hepatitis C virus infection: a population-based cohort study. J Viral Hepat 2012;19:272-277.

28 Liao C, Su T, Sung F, Chou W, Chen T: Does hepatitis C virus infection increase risk for stroke? A populationbased cohort study. PLoS One 2012;7:e31527.

29 Lee M, Yang H, Wang C, Jen C, Yeh S, Liu C, You S, Chen W, Chen C: Hepatitis C virus infection and increased risk of cerebrovascular disease. Stroke 2010;41:2894-2900.

30 Lee M, Yang H, Lu S, Jen C, You S, Wang L, Wang C, Chen W, Chen C, REVEAL HCV Study Group: Chronic hepatitis $\mathrm{C}$ virus infection increases mortality from hepatic and extrahepatic diseases: a community-based long-term prospective study. J Infect Dis 2012;206:469-477.

-31 Tomiyama H, Yamashina A, Arai T, Hirose K, Koji Y, Chikamori T, Hori S, Yamamoto Y, Dobe N, Hinohara S: Hepatitis C virus seropositivity, but not hepatitis B virus carrier or seropositivity, associated with increased pulse wave velocity. Atherosclerosis 2003;166:401-403.

-32 Ishizaka N, Ishizaka Y, Seki G, Nagai R, Yamakado M, Koike M: Association between hepatitis C virus core protein and carotid atherosclerosis. Circ J 2003;67:26-30.

-33 Mostafa A, Mohamed M, Saeed M, Hasan A, Fontanet A, Godsland I, Coady E, Esmat G, El-Hoseiny M, AbdulHamid M, Hughes A, Chaturvedi N: Hepatitis C infection and clearance: impact on atherosclerosis and cardiometabolic risk factors. Gut 2010;59:1135-1140.

-34 Adinolfi L, Restivo L, Zampino R, Adinolfi L, Restivo L, Zampino R, Guerrera B, Lonardo A, Ruggiero L, Riello F, Loria P, Florio A: Chronic HCV infection is a risk of atherosclerosis. Role of HCV and HCV-related steatosis. Atherosclerosis 2012;221:496-502.

35 Petta S, Torres D, Fazio G, Cammà C, Cabibi D, Di Marco V, Licata A, Marchesini G, Mazzola A, Parriniello G, Novo S, Licata G, Craxi A: Carotid atherosclerosis and chronic hepatitis C. a prospective study of risk associations. Hepatology 2012;55:1317-1323.

-36 Vassalle C, Masini S, Bianchi F, Zucchelli G: Evidence for association between hepatitis C virus seropositivity and coronary artery disease. Heart 2004;90:565-566.

37 Alyan O, Kacmaz F, Ozdemir O, Deveci B, Astan R, Celebi A, Ilkay E: Hepatitis C infection is associated with increased coronary artery atherosclerosis defined by modified Reardon severity score system. Circ J 2008;72:1960-1965. 


\section{Kidney \\ Blood Pressure Research}

-38 Younossi Z, Stepanova M, Nader F, Younossi Z, Elsheikh E: Associations of chronic hepatitis C with metabolic and cardiac outcomes. Aliment Pharmacol Ther 2013;37:647-652.

-39 Maruyama Y, Koda M, Oyake N, Sato H, Fujii Y, Horie Y, Murawaki Y: Myocardial injury in patients with chronic hepatitis C infection. J Hepatol 2013;58:11-15.

40 Yelken B, Gorgulu N, Caliskan Y, Elitok A, Cimen A, Yazici H, Oflaz H, Turkmen A, Sever M: Association between chronic hepatitis $\mathrm{C}$ infection and coronary flow reserve in dialysis patients with failed renal allografts. Transplant Proc 2009;41:1519-1523.

41 Oyake N, Shimada T, Murakami Y, Ishibashi Y, Satoh H, Suzuki K, Matsumori A, Oda T: Hepatitis C virus infection as a risk factor for increased aortic stiffness and cardiovascular events in dialysis patients. J Nephrol 2008;21:345-353.

-42 Caliskan Y, Oflaz H, Pusuroglu H, Boz H, Yazici H, Tamer S, Karsidag K, Yildiz A: Hepatitis C virus infection in hemodialysis patients is not associated with insulin resistance, inflammation and atheroslerosis. Clin Nephrol 2009;71:147-157.

43 Kalantar-Zadeh K, Kilpatrick R, McAllister C, Miller L, Daar E, Gjertson D, Kopple J, Greenland S: Hepatitis C virus and death risk in hemodialysis patients. J Am Soc Nephrol 2007;18:1584-1593.

44 Tsai H, Chen P, Liu C, Hung P, Chen M, Chiang C, Kao J, Hung K: Association of hepatitis C virus infection and malnutrition-inflammation complex syndrome in maintenance haemodialysis patients. Nephrol Dial Transplant 2012;27:1176-1183.

-45 Elsurer R, Afsar B, Sezer S, Arat Z, Ozdemir F, Haberal M: Malnutrition inflammation score is associated with coronary artery disease in hepatitis $\mathrm{C}$ virus-infected hemodialysis patients. Eur J Clin Nutrition 2008;62:1449-1454.

46 Hu K, Lee S, Hu S, Xia W, Hillebrand D, Kyulo N: Clinical presentation of chronic hepatitis C in patients with end-stage renal disease and on haemodialysis versus those with normal renal function. Am J Gastroenterol 2005;100:2010-2018.

-47 Wang S, Liu H, Chou C, Huang C, Shih C, Chen C: Mortality in hepatitis C-positive patients treated with peritoneal dialysis. Perit Dial Intern 2008;28:183-187.

48 Nascimento M, Suliman M, Bruchfeld A, Hayashi S, Manfro R, Qureshi A, Pecoits-Filho R, Pachaly M, Renner L, Stenvinkel P, Riella M, Lindholm B: The influence of hepatitis C and iron replacement therapy on plasma pentosidine levels in haemodialysis patients. Nephrol Dial Transplant 2004;19:3112-3116.

-49 Nascimento M, Bruchfeld A, Suliman M, Hayashi S, Pecoits-Filho R, Manfro R, Pachaly M, Renner L, Stenvinkel P, Riella M, Lindholm B: Effect of hepatitis C serology on C-reactive protein in a cohort of Brazilian hemodialysis patients. Braz J Med Biol Res 2005;38:783-788.

50 Arsov S, Graaff R, Morariu A, van Oeveren W, Smit A, Busletic I, Trajcevska L, Selim G, Dzekova P, Stegmayr B, Sikole A, Rakhorst G: Does hepatitis C increase the accumulation of advanced glycation end products in haemodialysis patients? Nephrol Dial Transplant 2010;25:885-891.

51 Liu C, Huang C, Liu C, Dai C, Liang C, Huang J, Hung P, Tsai H, Tsai M, Chen S, Lin J, Yang S, Su T, Yang H, Chen P, Chen D, Chuang W, Yu M, Kao J: Pegylated interferon- $\alpha 2$ a with or without low-dose ribavirin for treatment-naive patients with hepatitis $\mathrm{C}$ virus genotype 1 receiving hemodialysis: a randomized trial. Ann Intern Med 2013;159:729-738.

-52 Fabrizi F, Dixit V, Messa P, Martin P: Antiviral therapy (pegylated interferon plus ribavirin) of hepatitis C in dialysis patients: Meta-analysis of clinical studies. J Viral Hepat 2014, DOI 10.1111/jvh.12276.

53 Aghemo A, De Francesco R: New horizons in hepatitis C antiviral therapy with direct-acting antivirals. Hepatology 2013;58:428-438.

54 Baugh J, Garcia-Rivera J, Gallay P: Host-targeting agents in the treatment of hepatitis C: A beginning and an end? Antiviral Res 2013;100:555-561.

55 Dumortier J, Guillaud O, Gagnieu M, Janbob B, Juillard L, Morelon E, Leroy V: Antiviral triple therapy with telaprevir in hemodialysed patients: is it feasible? J Clin Virol 2013;56:146-149. 